\title{
Editorial
}

\section{Standard Nomenclature for Renal Replacement Therapy in Acute Kidney Injury: Very Much Needed!}

\section{Claudio Ronco}

Department of Nephrology, Dialysis and Transplantation, International Renal Research Institute of Vicenza (IRRIV), San Bortolo Hospital, Vicenza, Italy

Practice patterns in patients with acute kidney injury (AKI) requiring renal support or renal replacement are strongly affected by the results of trials whose value is often limited by a relatively small number of patients or an underpowered design. For this reason, registries and large databases are frequently used as a surrogate although database cleaning is often challenging or even impossible. All these barriers to a useful utilization of large data systems are jeopardized by the lack of a standard for the terminology used. At the same time, the management of critically ill patients with AKI who need renal replacement therapy (RRT) requires a multidisciplinary approach. When nephrologists, intensivists and nurses gather at the bedside of critically ill patients to decide on treatment strategies and to execute treatment decisions, it would be important for them to be able to rely on a common language and terminology. In contrast, the terminology used to describe the different modalities of RRT and the terms assigned to various parameters are often confusing and continually changing. A consensus nomenclature useful to homogenize this complex field and aiming at attaining a shared language for all parties involved is very much needed. Assuming all players reach an agreement on terminology and nomenclature, we can expect that any new development from industry will adopt this standard terminology to facilitate comprehension, progress and the possibility to share collected data in large databases.

\section{KARGER}

(c) 2014 S. Karger AG, Basel

0253-5068/14/0382-000I\$39.50/0

E-Mail karger@karger.com

www.karger.com/bpu
ADQI (Acute Dialysis Initiative) has traditionally led the field when it comes to changes in the treatment of AKI and continuous renal replacement therapies (CRRT), and also, in this case, a future consensus conference on the important contribution of information communication technology and large data management is in the planning phase. As a prerequisite for this consensus, the adoption of a common terminology would become quintessential. The area that should be covered are the basic principles underlying RRT technologies and the application of those principles to direct patient care, the principles of solute and fluid transport mechanisms across membranes and the mechanical aspects of the devices utilized to provide RRT. Other aspects should include basic aspects and characteristics of continuous renal replacement therapies (CRRT), which are most often utilized in the treatment of critically ill patients with severe AKI.

A consensus nomenclature should be suggested for parameters of new hardware and devices. CRRT 'hardware' includes the machine and its dedicated disposables. Knowledge of the nomenclature and the functions of the whole machine and its main components is extremely important, not only for the nurses or technicians who are the main users of the device but also for the clinicians who issue prescriptions and manage patient care. A standardization should be encouraged as far as hardware and software are concerned such as screen and visual interface, light and buzzer indicators, alarms, pressure sensors and pres- 
sure identification, pumps and flows (possibly color-coded in graphs), fluid control systems and related parameters, dose prescription and delivery parameters, anticoagulant regime, machine procedure phases (preparation, priming, connection to patient, extracorporeal circulation, special procedures, flushing and blood restitution). Similarly, standard names should be used for disposables such as filters and lines defining the permeability (low flux, high flux, etc.) and the function (ultrafilter, dialyzer, hemofilter and hemodiafilter). Attention should similarly be paid to the classification of the components of fluid balance and fluid balance error. In particular, it should be paid to define different modalities (intermittent, continuous and hybrid) and their scope (ultrafiltration, dialysis, hemofiltration, hemodiafiltration, plasmapheresis, adsorption hemoperfusion, plasma filtration adsorption, etc.). Treatments should also be defined depending on the therapeutic intent (heart support, liver support, fluid removal, extracorporeal $\mathrm{CO}_{2}$ removal, etc.).
In conclusion, when faced with a complex patient, practitioners can use a growing range of extracorporeal treatment options [1]. Understanding the importance of giving identical parameters identical names will certainly encourage the creation of a new standard nomenclature that will make it possible to use all collected data to build large registries that should yield useful information for treating patients. This should be the work to focus on during the months leading up to the ADQI consensus conference on information communication technology and large databases.

Reference

1 Ronco C: Acute kidney injury and renal replacement therapy: ADOPPS wanted! Blood Purif 2014;37:I-II. 